\title{
Simultaneous estimation of genetic linkage and preferential pairing factor for a triploid population with unphased markers
}

\author{
WANG JianXin ${ }^{1,2}$, DONG XiaoLi ${ }^{1}$, PANG XiaoMing ${ }^{2,3,4}$, LÜ YaFei $^{2}$, YI HuaLin ${ }^{5}$, \\ YANG XiaoXia ${ }^{2}$, WANG Zhong ${ }^{2,6}$, WU Song ${ }^{7} \&$ WU RongLing ${ }^{2,3,4,6^{*}}$ \\ ${ }^{1}$ School of Information, Beijing Forestry University, Beijing 100083, China; \\ ${ }^{2}$ Center for Computational Biology, Beijing Forestry University, Beijing 100083, China; \\ ${ }^{3}$ National Engineering Laboratory for Tree Breeding, Beijing Forestry University, Beijing 100083, China; \\ ${ }^{4}$ Key Laboratory of Genetics and Breeding in Forest Trees and Ornamental Plants, Beijing Forestry University, Beijing 100083, China; \\ ${ }^{5}$ The National Key Laboratory for Crop Genetic Improvement, Huazhong Agricultural University, Wuhan 430070, China; \\ ${ }^{6}$ Center for Statistical Genetics, The Pennsylvania State University, Hershey, PA 17033, USA; \\ ${ }^{7}$ Department of Applied Mathematics \& Statistics, State University of New York, Stony Brook, NY 11794, USA
}

Received February 24, 2012; accepted April 17, 2012; published online May 19, 2012

\begin{abstract}
Triploids, recognized to occur more frequently in natural and experimental populations of many species than previously appreciated, display important economic and biological values. Despite this, however, linkage analysis for triploids has not been well explored. We develop a statistical model for estimating and testing the linkage between molecular markers in a triploid population derived from a tetraploid and diploid parent. The model incorporates one important meiotic feature of tetraploids by which more homologous chromosomes pair with a greater likelihood than less homologous chromosomes. By implementing the EM algorithm within the maximum likelihood framework, the model provides a procedure for simultaneous estimation of the linkage and preferential pairing factor. The model accommodates the segregating patterns of pseudotest markers and intercross markers with different amounts of informativeness. The utility of the model was validated through a real data analysis and simulation studies. The model provides a statistical tool for linkage analysis in a triploid population by taking into account meiotic behavior of tetraploids. Results from the model will help to shed light on the genetic diversity and origin of a polyploid population.
\end{abstract}

triploid, tetraploid, linkage, preferential pairing factor, outcrossing species

Citation: Wang J X, Dong X L, Pang X M, et al. Simultaneous estimation of genetic linkage and preferential pairing factor for a triploid population with unphased markers. Chin Sci Bull, 2012, 57: 2711-2720, doi: 10.1007/s11434-012-5217-9

Triploids are an organism that has three complete sets of chromosomes, i.e., three times the haploid number of chromosomes in the cell nucleus. It has been recognized that the occurrence of triploids in natural populations is more prevalent than previously appreciated [1]. Because of the extra set of chromosomes leading to an increase in cell nucleus dimensions, triploids often display more vigorous vegetative growth than their diploid counterparts [2-4]. Hence, for those plants whose vegetative parts are economically useful, triploids can be of commercial use. In forest trees, selection

*Corresponding author (email: rwu@phs.psu.edu) for superior triploids as an important breeding strategy has been stimulated by Nilson-Ehle's [5] first discovery of a giant aspen triploid $[1,6,7]$. Nowadays, a growing body of biotechnologies has been initiated to unravel the genetic control and regulation of triploids [8], ultimately accelerating the efficiency of plant breeding for these special organisms.

As a powerful tool, genetic mapping has now been used routinely to study the structure and variation of genomes related to complex phenotypes. For a diploid plant, statistical models for genetic mapping with molecular markers have been well developed in the literature [9]. Despite the availability of several models for genetic mapping in poly- 
ploids [10-12], there is still significant inadequacy of mapping models and algorithms that incorporate the formation mechanisms and inheritance of polyploids. The purpose of this study is to develop a statistical model for linkage analysis with molecular markers in a triploid population derived from an outcrossing tetraploid and an outcrossing diploid parent. In Populus, crossing a diploid $P$. tremuloides female with a tetraploid male $P$. tremula has produced triploid progeny $[13,14]$. A similar phenomenon was observed in other species, such as Citrus [15].

To study the segregation of markers in a triploid population, we need to first know how the outcrossing tetraploid and diploid parents transmit their alleles into the progeny. Although the inheritance of a diploid can be readily modeled, a special consideration will be needed to model the complexity of inheritance characterized by a tetraploid. Based on the pattern of chromosomal pairings, polyploids can be classified into bivalent polyploids, in which only two chromosomes pair at a time, and multivalent polyploids, in which more than two chromosomes pair during meiosis [16]. Based on the pattern of how chromosomes pair at meiosis, specific models have been developed for the linkage analysis of molecular markers in bivalent polyploids [12] and multivalent polyploids $[16,17]$. In this article, we will incorporate the bivalent inheritance into a linkage model for a triploid progeny population derived from a tetraploid and diploid. We will first describe the model for analyzing fully informative markers and then extend the model to consider the linkage of dominant markers. Simulation studies are carried out to test the statistical properties of the model. We use a real data set for citrus to validate the utilization of the model. The new model provides a means for constructing genetic linkage maps for QTL mapping in a triploid progeny population.

\section{The model}

\subsection{Preferential pairing factor}

Consider a bivalent tetraploid plant derived from the chromosomal combination of distinct genomes and subsequent chromosomal doubling [18]. Four sets of chromosomes for the tetraploid are labelled as $\mathbf{1}, \mathbf{2}, \mathbf{3}$, and $\mathbf{4}$, respectively. There are three different homologous patterns:

I. Chromosomes $\mathbf{1}$ and $\mathbf{2}$ are homologous while chromosomes $\mathbf{3}$ and $\mathbf{4}$ are homologous. Chromosomes from different homologous pairs, i.e., $\mathbf{1}$ and $\mathbf{3}, \mathbf{1}$ and $\mathbf{4 , 2}$ and $\mathbf{3}$, and $\mathbf{2}$ and $\mathbf{4}$, are homoeologous.

II. Chromosomes $\mathbf{1}$ and $\mathbf{3}$ and, therefore, chromosomes $\mathbf{2}$ and $\mathbf{4}$ are homologous. Chromosome pairs, $\mathbf{1}$ and $\mathbf{2 , 1}$ and $\mathbf{4 ,}$ $\mathbf{2}$ and $\mathbf{3}$, and $\mathbf{3}$ and $\mathbf{4}$, are homoeologous.

III. Chromosomes $\mathbf{1}$ and $\mathbf{4}$ and, therefore, chromosomes $\mathbf{2}$ and $\mathbf{3}$ are homologous. Chromosome pairs, $\mathbf{1}$ and $\mathbf{2 , 1}$ and $\mathbf{3}$, $\mathbf{2}$ and $\mathbf{4}$, and $\mathbf{3}$ and $\mathbf{4}$, are homoeologous.

The affinity of chromosomal pairing may be higher be- tween homologous pairs than between the homoeologues [19]. Two-third of this difference is defined as the preferential pairing factor $(p)$ [20]. By considering all possible pairs, i.e., $\mathbf{1}$ pairs with $\mathbf{2}$, then $\mathbf{3}$ must pair $\mathbf{4}$; if 1 pairs with $\mathbf{3}$, then 2 must pair 4 ; if 1 pairs 4 , then 2 must pair 3 . Of the three possibilities, the first is homologous and the rest are homoeologous for the first configuration. The homologous combination has a probability of $\theta_{1}=1 / 3+p$, and the two homoeologous combinations each have a probability of $\theta_{2}=$ $1 / 3-1 / 2 p$. If $p=2 / 3$, then homoeologous chromosomes do not pair, i.e., chromosomal pairings happen strictly between the homologues. When $p=0$, then all the four chromosomes are homologous, and they will pair randomly. Thus, the value of $p$ ranges from 0 to $2 / 3$ [20].

\subsection{Gametic model}

Consider a heterozygous bivalent tetraploid line crossed with a heterozygous diploid line to generate a triploid hybrid population of size $n$. There are three types of markers that are segregating in this population: (1) pseudotest markers that are heterozygous in the diploid parent but homozygous in the tetraploid parent; (2) pseudotest markers that are heterozygous in the tetraploid parent but homozygous in the diploid parent, and (3) inter-cross markers that are heterozygous in both parents. A traditional backcross model [9] can be used to analyze the first type of markers. However, we will need to develop new models for analyzing the last two types of markers based on meiotic features of a tetraploid. For the second types of markers, the genotypes of progeny are consistent with the genotypes of gametes produced by the heterozygous tetraploid parent and, therefore, the derivation of mapping models can be based on the segregation of gametes.

(i) Fully information markers. The marker at which a different allele is carried by each single chromosome is called fully informative marker [12]. For example, a fully informative marker has four different alleles, expressed as $1,2,3$ and 4 on chromosomes $\mathbf{1}, \mathbf{2}, \mathbf{3}$, and $\mathbf{4}$, respectively, for an outcrossing tetraploid and, thus, the genotype of this tetraploid is denoted as 1234. The genotype of two fully informative markers for the tetraploid is expressed as 1234/1234. Assume that these two markers, linked with a recombination fraction of $r$, have a diplotype \begin{tabular}{ll|l|l|l}
1 & 2 & 3 & 4 \\
& 1 & 2 & 3 & 4
\end{tabular} \mid . For each marker, the types of gametes produced by the tetraploid depend on chromosomal pairings. If the homologous chromosomes pair, i.e., chromosome 1 pairs with $\mathbf{2}$ and, therefore, chromosome $\mathbf{3}$ pairs with $\mathbf{4}$ for the first homologous configuration, then haploid gametes at a marker produced by the first pair are 1 and 2 and those produced by the second pair are 3 and 4, leading to diploid gametes 13, 14, 23 , and 24 . It can be seen that the probability of producing these four gametes is $\theta_{1}$ for the heterozygous tetraploid. 
Similarly, we can find that the two types of homoeologous-chromosomal pairings will produce, at a probability of $\theta_{2}$, diploid gametes $12,14,23$, and 34 as well as 12, 13, 24, and 34 , respectively. Overall, the tetraploid produces $(6 \times 6)=$ 36 diploid gametes for the two markers, whose frequencies are derived for homologous pattern I, II, or III (Table 1). We will develop a gametic model for estimating the recombination fraction and preferential pairing factor under each of these three homologous patterns for a triploid progeny population.

Let $n_{j_{1} j_{2} / k_{1} k_{2}}$ denote the observation of diploid gamete $j_{1} j_{2} / k_{1} k_{2}$, where $j_{1}<j_{2}=1,2,3,4$ are the alleles of the first marker and $k_{1}<k_{2}=1,2,3,4$ are the alleles of the second marker. Based on the gamete frequencies given for diplotype \begin{tabular}{l|l|l|l|l}
1 & 2 & 3 & 4 & \\
& 1 & 2 & 3 & 4
\end{tabular} \mid under homologous pattern I (Table 1), we formulate a polynomial likelihood from which to solve the recombination fraction and preferential pairing factor by

$$
\begin{gathered}
r=\frac{1}{2 n}\left[n+\left(n_{12 / 34}+n_{13 / 24}+n_{14 / 23}+n_{23 / 14}+n_{24 / 13}+n_{34 / 12}\right)\right. \\
\left.-\left(n_{12 / 12}+n_{13 / 13}+n_{14 / 14}+n_{23 / 23}+n_{24 / 24}+n_{34 / 34}\right)\right], \\
p=\frac{2}{3}\left[\frac{n_{1} \phi_{1}+n_{2} \phi_{2}}{n_{1} \phi_{1}+n_{2} \phi_{2}+n-n_{1}-n_{2}}\right],
\end{gathered}
$$

where

$$
\begin{gathered}
\phi_{1}=\frac{p}{\frac{1}{3}+p}, \\
\phi_{2}=\frac{\frac{p}{2}}{\frac{2}{3}+\frac{p}{2}},
\end{gathered}
$$

with $n_{1}=n_{13 / 14}+n_{13 / 23}+n_{14 / 13}+n_{14 / 24}+n_{23 / 13}+n_{23 / 14}+n_{24 / 14}+$ $n_{24 / 23}, n_{2}=n_{13 / 13}+n_{13 / 24}+n_{14 / 14}+n_{14 / 23}+n_{23 / 14}+n_{23 / 23}+n_{24 / 13}+$ $n_{24 / 24}$. The estimation of $r$ is straightforward using the analytical expression (1). Howerer, we need to use a numerical approach for estimating $p$ by iterating between equations (3) and (4) and equation (2).

For an outcrossing tetraploid parent, its homologous pattern is unknown, but we can derive similar equations to estimate $r$ and $p$ under homologous patterns II and III (based on Table 1), respectively. Furthermore, when two fully informative markers are considered, we will have a total of 4 !

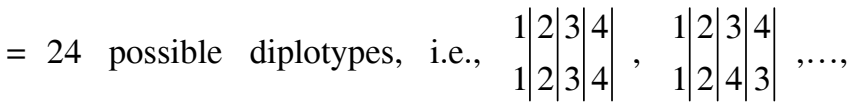
\begin{tabular}{l|l|l|l|l|l}
1 & 2 & 3 & 4 & \\
4 & 3 & 2 & 1
\end{tabular} \mid . By considering all possible combinations of three homologous patterns (Table 1) and 24 diplotypes, we cal- culate parameters $r$ and $p$ from which the likelihood is estimated. The combination that gives the largest likelihood is the most likely homologous pattern and diplotype. The optimal estimates of these two parameters should be derived from the most likely combination.

The hypothesis about the linkage between the two markers is formulated as

$$
\begin{aligned}
& H_{0}: r=0.5, \\
& H_{1}: r \neq 0.5 .
\end{aligned}
$$

The log-likelihood ratio under the null and alternative hypotheses is calculated which follows a $\chi^{2}$-distribution with one degree of freedom.

We can also test whether the preferential pairing factor $p$ is equal to $2 / 3$ (only homologous chromosomes pairs) and 0 (there is no preference in chromosomal pairing). For each test, a log-likelihood ratio is calculated and compared with the critical threshold $\chi^{2}$ value with one degree of freedom.

(ii) Partially information markers. The marker at which the same alleles are carried by different chromosomes is called partially informative marker [12]. The examples of partially informative markers include genotypes 1122,1222 , and 1233 for a tetraploid. Let us consider a pair of partially informative markers with genotypes 1222 and 1233, respectively, which are linked with recombination fraction $r$. They

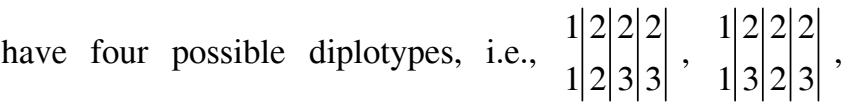

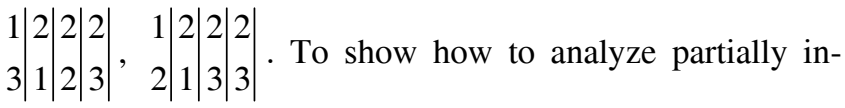
formative markers, let us consider the first diplotype. This diplotype may have two possible homologous patterns I and II or III whose diploid gamete frequencies can be derived (given in Table 2) by collapsing the cells of the same gamete genotypes.

Based on Table 2, we derived analytical solutions to estimate the recombination fraction and preferential pairing factor under homologous pattern I, expressed as

$$
\begin{aligned}
& r=\frac{1}{n}\left(n_{12 / 23}+n_{12 / 33}+n_{22 / 12}+n_{22 / 13}\right), \\
& \theta_{2}=\frac{1}{n}\left(n_{12 / 12}+n_{12 / 33}+n_{22 / 12}+n_{22 / 33}\right) .
\end{aligned}
$$

The estimate of the preferential pairing factor is obtained by $p=\frac{1}{3}\left(2-6 \theta_{2}\right)$. The estimates of $r$ and $p$ under homologous pattern II or I are based on the following procedure:

$$
\begin{gathered}
r=\frac{1}{n}\left(n_{12 / 23}+n_{12 / 33}+n_{22 / 12}+n_{22 / 13}\right), \\
p=\frac{1}{n} \phi\left(n_{12 / 12}+n_{12 / 33}+n_{22 / 12}+n_{22 / 33}\right),
\end{gathered}
$$




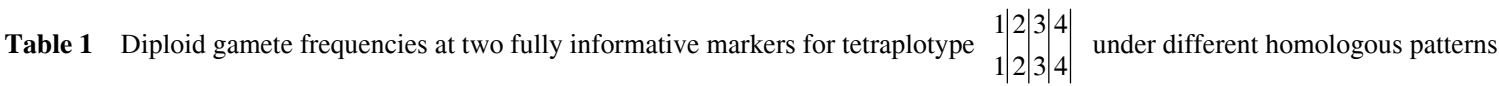

\begin{tabular}{|c|c|c|c|c|c|c|}
\hline \multirow{2}{*}{$\begin{array}{c}\text { Marker } \\
1\end{array}$} & \multicolumn{6}{|c|}{ Marker 2} \\
\hline & 12 & 13 & 14 & 23 & 24 & 34 \\
\hline \multicolumn{7}{|c|}{ Homologous pattern I } \\
\hline 12 & $\frac{1}{2}(1-r)^{2} \theta_{2}$ & $\frac{1}{4} r(1-r) \theta_{2}$ & $\frac{1}{4} r(1-r) \theta_{2}$ & $\frac{1}{4} r(1-r) \theta_{2}$ & $\frac{1}{4} r(1-r) \theta_{2}$ & $\frac{1}{2} r^{2} \theta_{2}$ \\
\hline 13 & $\frac{1}{4} r(1-r) \theta_{2}$ & $\frac{1}{4}(1-r)^{2}\left(\theta_{1}+\theta_{2}\right)$ & $\frac{1}{4} r(1-r) \theta_{1}$ & $\frac{1}{4} r(1-r) \theta_{1}$ & $\frac{1}{4} r^{2}\left(\theta_{1}+\theta_{2}\right)$ & $\frac{1}{4} r(1-r) \theta_{2}$ \\
\hline 14 & $\frac{1}{4} r(1-r) \theta_{2}$ & $\frac{1}{4} r(1-r) \theta_{1}$ & $\frac{1}{4}(1-r)^{2}\left(\theta_{1}+\theta_{2}\right)$ & $\frac{1}{4} r^{2}\left(\theta_{1}+\theta_{2}\right)$ & $\frac{1}{4} r(1-r) \theta_{1}$ & $\frac{1}{4} r(1-r) \theta_{2}$ \\
\hline 23 & $\frac{1}{4} r(1-r) \theta_{2}$ & $\frac{1}{4} r(1-r) \theta_{1}$ & $\frac{1}{4} r^{2}\left(\theta_{1}+\theta_{2}\right)$ & $\frac{1}{4}(1-r)^{2}\left(\theta_{1}+\theta_{2}\right)$ & $\frac{1}{4} r(1-r) \theta_{1}$ & $\frac{1}{4} r(1-r) \theta_{2}$ \\
\hline 24 & $\frac{1}{4} r(1-r) \theta_{2}$ & $\frac{1}{4} r^{2}\left(\theta_{1}+\theta_{2}\right)$ & $\frac{1}{4} r(1-r) \theta_{1}$ & $\frac{1}{4} r(1-r) \theta_{1}$ & $\frac{1}{4}(1-r)^{2}\left(\theta_{1}+\theta_{2}\right)$ & $\frac{1}{4} r(1-r) \theta_{2}$ \\
\hline 34 & $\frac{1}{2} r^{2} \theta_{2}$ & $\frac{1}{4} r(1-r) \theta_{2}$ & $\frac{1}{4} r(1-r) \theta_{2}$ & $\frac{1}{4} r(1-r) \theta_{2}$ & $\frac{1}{4} r(1-r) \theta_{2}$ & $\frac{1}{2}(1-r)^{2} \theta_{2}$ \\
\hline \multicolumn{7}{|c|}{ Homologous pattern II } \\
\hline 12 & $\frac{1}{4}(1-r)^{2}\left(\theta_{1}+\theta_{2}\right)$ & $\frac{1}{4} r(1-r) \theta_{2}$ & $\frac{1}{4} r(1-r) \theta_{1}$ & $\frac{1}{4} r(1-r) \theta_{1}$ & $\frac{1}{4} r(1-r) \theta_{2}$ & $\frac{1}{4} r^{2}\left(\theta_{1}+\theta_{2}\right)$ \\
\hline 13 & $\frac{1}{4} r(1-r) \theta_{2}$ & $\frac{1}{2}(1-r)^{2} \theta_{2}$ & $\frac{1}{4} r(1-r) \theta_{2}$ & $\frac{1}{4} r(1-r) \theta_{2}$ & $\frac{1}{2} r^{2} \theta_{2}$ & $\frac{1}{4} r(1-r) \theta_{2}$ \\
\hline 14 & $\frac{1}{4} r(1-r) \theta_{1}$ & $\frac{1}{4} r(1-r) \theta_{2}$ & $\frac{1}{4}(1-r)^{2}\left(\theta_{1}+\theta_{2}\right)$ & $\frac{1}{4} r^{2}\left(\theta_{1}+\theta_{2}\right)$ & $\frac{1}{4} r(1-r) \theta_{2}$ & $\frac{1}{4} r(1-r) \theta_{1}$ \\
\hline 23 & $\frac{1}{4} r(1-r) \theta_{1}$ & $\frac{1}{4} r(1-r) \theta_{2}$ & $\frac{1}{4} r^{2}\left(\theta_{1}+\theta_{2}\right)$ & $\frac{1}{4}(1-r)^{2}\left(\theta_{1}+\theta_{2}\right)$ & $\frac{1}{4} r(1-r) \theta_{2}$ & $\frac{1}{4} r(1-r) \theta_{1}$ \\
\hline 24 & $\frac{1}{4} r(1-r) \theta_{2}$ & $\frac{1}{2} r^{2} \theta_{2}$ & $\frac{1}{4} r(1-r) \theta_{2}$ & $\frac{1}{4} r(1-r) \theta_{2}$ & $\frac{1}{4}(1-r)^{2} \theta_{2}$ & $\frac{1}{4} r(1-r) \theta_{2}$ \\
\hline 34 & $\frac{1}{4} r^{2}\left(\theta_{1}+\theta_{2}\right)$ & $\frac{1}{4} r(1-r) \theta_{2}$ & $\frac{1}{4} r(1-r) \theta_{1}$ & $\frac{1}{4} r(1-r) \theta_{1}$ & $\frac{1}{4} r(1-r) \theta_{2}$ & $\frac{1}{4}(1-r)^{2}\left(\theta_{1}+\theta_{2}\right)$ \\
\hline \multicolumn{7}{|c|}{ Homologous pattern III } \\
\hline 12 & $\frac{1}{4}(1-r)^{2}\left(\theta_{1}+\theta_{2}\right)$ & $\frac{1}{4} r(1-r) \theta_{1}$ & $\frac{1}{4} r(1-r) \theta_{2}$ & $\frac{1}{4} r(1-r) \theta_{2}$ & $\frac{1}{4} r(1-r) \theta_{1}$ & $\frac{1}{4} r^{2}\left(\theta_{1}+\theta_{2}\right)$ \\
\hline 13 & $\frac{1}{4} r(1-r) \theta_{1}$ & $\frac{1}{4}(1-r)^{2}\left(\theta_{1}+\theta_{2}\right)$ & $\frac{1}{4} r(1-r) \theta_{2}$ & $\frac{1}{4} r(1-r) \theta_{2}$ & $\frac{1}{4} r^{2}\left(\theta 1+\theta_{2}\right)$ & $\frac{1}{4} r(1-r) \theta_{1}$ \\
\hline 14 & $\frac{1}{4} r(1-r) \theta_{2}$ & $\frac{1}{4} r(1-r) \theta_{2}$ & $\frac{1}{2}(1-r)^{2} \theta_{2}$ & $\frac{1}{2} r^{2} \theta_{2}$ & $\frac{1}{4} r(1-r) \theta_{2}$ & $\frac{1}{4} r(1-r) \theta_{2}$ \\
\hline 23 & $\frac{1}{4} r(1-r) \theta_{2}$ & $\frac{1}{4} r(1-r) \theta_{2}$ & $\frac{1}{2} r^{2} \theta_{2}$ & $\frac{1}{2}(1-r)^{2} \theta_{2}$ & $\frac{1}{4} r(1-r) \theta_{2}$ & $\frac{1}{4} r(1-r) \theta_{2}$ \\
\hline 24 & $\frac{1}{4} r(1-r) \theta_{1}$ & $\frac{1}{4} r^{2}\left(\theta_{1}+\theta_{2}\right)$ & $\frac{1}{4} r(1-r) \theta_{2}$ & $\frac{1}{4} r(1-r) \theta_{2}$ & $\frac{1}{4}(1-r)^{2}\left(\theta_{1}+\theta_{2}\right)$ & $\frac{1}{4} r(1-r) \theta_{1}$ \\
\hline 34 & $\frac{1}{4} r^{2}\left(\theta_{1}+\theta_{2}\right)$ & $\frac{1}{4} r(1-r) \theta_{1}$ & $\frac{1}{4} r(1-r) \theta_{2}$ & $\frac{1}{4} r(1-r) \theta_{2}$ & $\frac{1}{4} r(1-r) \theta_{1}$ & $\frac{1}{4}(1-r)^{2}\left(\theta_{1}+\theta_{2}\right)$ \\
\hline
\end{tabular}

where

$$
\phi_{2}=\frac{\frac{p}{2}}{\frac{2}{3}+\frac{p}{2}}
$$

\subsection{Zygotic model}

For intercross markers, both parents are heterozygous so that the segregation pattern of progeny genotypes is differ- ent from that of gamete genotypes. Consider two fully informative markers with alleles $1,2,3$, and 4 for the tetraploid parent and alleles 5 and 6 for the diploid parent. Let us first assume the diplotype of the diplotype parent as \begin{tabular}{l|l|l|l}
1 & 2 & 3 & 4 \\
1 & 2 & 3 & 4
\end{tabular} \mid and the diplotype of the diplotype parent as $5|6| 6 \mid$. The frequencies of 36 diploid gametes by the tetraploid parent under different homologous patterns are given in Table 1. It is easy to get the frequencies of four haploid gametes by the diploid parent as 


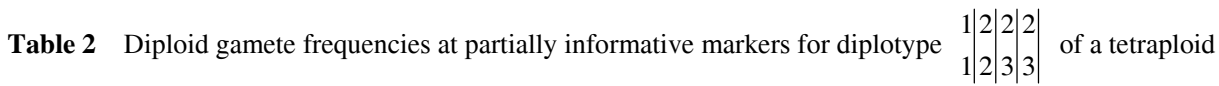

\begin{tabular}{|c|c|c|c|c|}
\hline \multirow{2}{*}{$\begin{array}{c}\text { Marker } \\
1\end{array}$} & \multicolumn{4}{|c|}{ Marker 2} \\
\hline & 12 & 13 & 23 & 33 \\
\hline \multicolumn{5}{|c|}{ Homologous pattern I } \\
\hline 12 & $\frac{1}{2}(1-r) \theta_{2}$ & $\frac{1}{2}(1-r)\left(\theta_{1}+\theta_{2}\right)$ & $\frac{1}{2} r\left(\theta_{1}+\theta_{2}\right)$ & $\frac{1}{2} r \theta_{2}$ \\
\hline 22 & $\frac{1}{2} r \theta_{2}$ & $\frac{1}{2} r\left(\theta_{1}+\theta_{2}\right)$ & $\frac{1}{2}(1-r)\left(\theta_{1}+\theta_{2}\right)$ & $\frac{1}{2}(1-r) \theta_{2}$ \\
\hline 12 & $\frac{1}{4}(1-r)\left(\theta_{1}+\theta_{2}\right)$ & $\frac{1}{4}(1-r)\left(\theta_{1}+3 \theta_{2}\right)$ & $\frac{1}{4} r\left(\theta_{1}+3 \theta_{2}\right)$ & $\frac{1}{4} r\left(\theta_{1}+\theta_{2}\right)$ \\
\hline 22 & $\frac{1}{4} r\left(\theta_{1}+\theta_{2}\right)$ & $\frac{1}{4} r\left(\theta_{1}+3 \theta_{2}\right)$ & $\frac{1}{4}(1-r)\left(\theta_{1}+3 \theta_{2}\right)$ & $\frac{1}{4}(1-r)\left(\theta_{1}+\theta_{2}\right)$ \\
\hline
\end{tabular}

\begin{tabular}{c|cc} 
& 5 & 6 \\
\hline 5 & $\frac{1}{2}(1-r)$ & $\frac{1}{2} r$ \\
6 & $\frac{1}{2} r$ & $\frac{1}{2}(1-r)$
\end{tabular}.

Let $\mathbf{P}_{6 \times 6}$ denote the matrix for the frequencies of diploid gametes produced by the tetraploid parent and $\mathbf{Q}_{2 \times 2}$ denote the matrix for the frequencies of haploid gametes produced by the diploid parent. Thus, the frequencies of zygotic genotypes produced by the two heterozygous parents are expressed as

$$
\mathbf{P}_{6 \times 6} \otimes \mathbf{Q}_{2 \times 2}
$$

which is a $12 \times 12$ triplotype matrix (Table 3 ). From this matrix, we can derive an analytic solution to estimate the recombination fraction, but will need to implement the EM algorithm to estimate the preferential pairing factor. Since the tetraplotype of the tetraploid parent and diplotype of the diploid parent are unknown, we will need to incorporate a model selection approach for determining an optimal combination of tetraplotype and diplotype.

For partially informative markers, i.e., those at which chromosomes of the parents share the same alleles, the $12 \times 12$ triplotype matrix above will be collapsed into a matrix of reduced dimension because some triplotypes have the same triploid genotype. Table 4 lists the genotype frequencies of triploid progeny for two partially informative markers derived from outcrossing tetraploid and diploid parents. The tetraploid is assumed to have a tetraplotype \begin{tabular}{ll|l|l|l|l}
1 & 2 & 3 & 4 & \\
& 1 & 2 & 3 & 4 &
\end{tabular} and follow homologous pattern I. For any partially informative markers, we can implement an algorithm (including the EM algorithm) to estimate the recombination fraction and preferential pairing factor.

\section{Results}

\subsection{A worked example}

The model is demonstrated by analyzing a genetic data set collected for a triploid mapping population in Citrus. The study population of 79 triploid trees was derived from hybridization between an allotetraploid male (HR) and a diploid hybrid female (IB) [15]. The HR is a somatic hybrid between a Citrus sinensis Osbeck variety $(\mathrm{Hm})$ and a $C$. jambhiri Luss variety (RL), whereas the IB is a sexual hybrid between $C$. reticulata Blanco and $C$. ichangensis Swingle. During the past decades, dominant marker technologies, because of their fast and inexpensive advantages, have been widely used for the genetic mapping of underrepresented species including polyploids and trees [21]. In this mapping study, the diploid Hm, diploid RL, tetraploid HR, diploid IB, and triploid progeny were genotyped for PCR-based RAPD dominant markers. Based on the genotypes of the parents and original parents, all the segregating markers are classified into seven types (Table 5). For types 1-3, the pattern of segregation is identical for diploid gametes and triploid zygotes, but this is different for types 4-6 so that the estimation of their $r$ and $p$ should be based on triploid genotypes. Type 7 segregates according to a diploid marker which will not be considered here.

Let us first consider marker types 1-3. Types 1 and 2 each have two subtypes, depending on the number of dominant alleles in the original heterozygous diploid parents, Hm or RL. Together, we will need to develop a procedure for linkage analysis between five different types of markers, i.e., 1100, 1000, 0011, 0010, and 1010, where the first two alleles of a genotype are derived from the $\mathrm{Hm}$ parent and the second two from the RL parent. Whenever we have a marker that belongs to type 1 or 2 , we need to determine the most likely subtype. This can be done by calculating the likelihood under each subtype and choosing one with the largest likelihood.

To describe this procedure, we conduct linkage analysis for two markers both from type 1 . Then it is possible to have four combinations of markers, 1100/1100, 1100/1000, $1000 / 1100$, and 1000/1000. The first two alleles at each marker are from the $\mathrm{Hm}$ parent, whereas the second two alleles are from the RL parent. Thus, it is reasonable to 


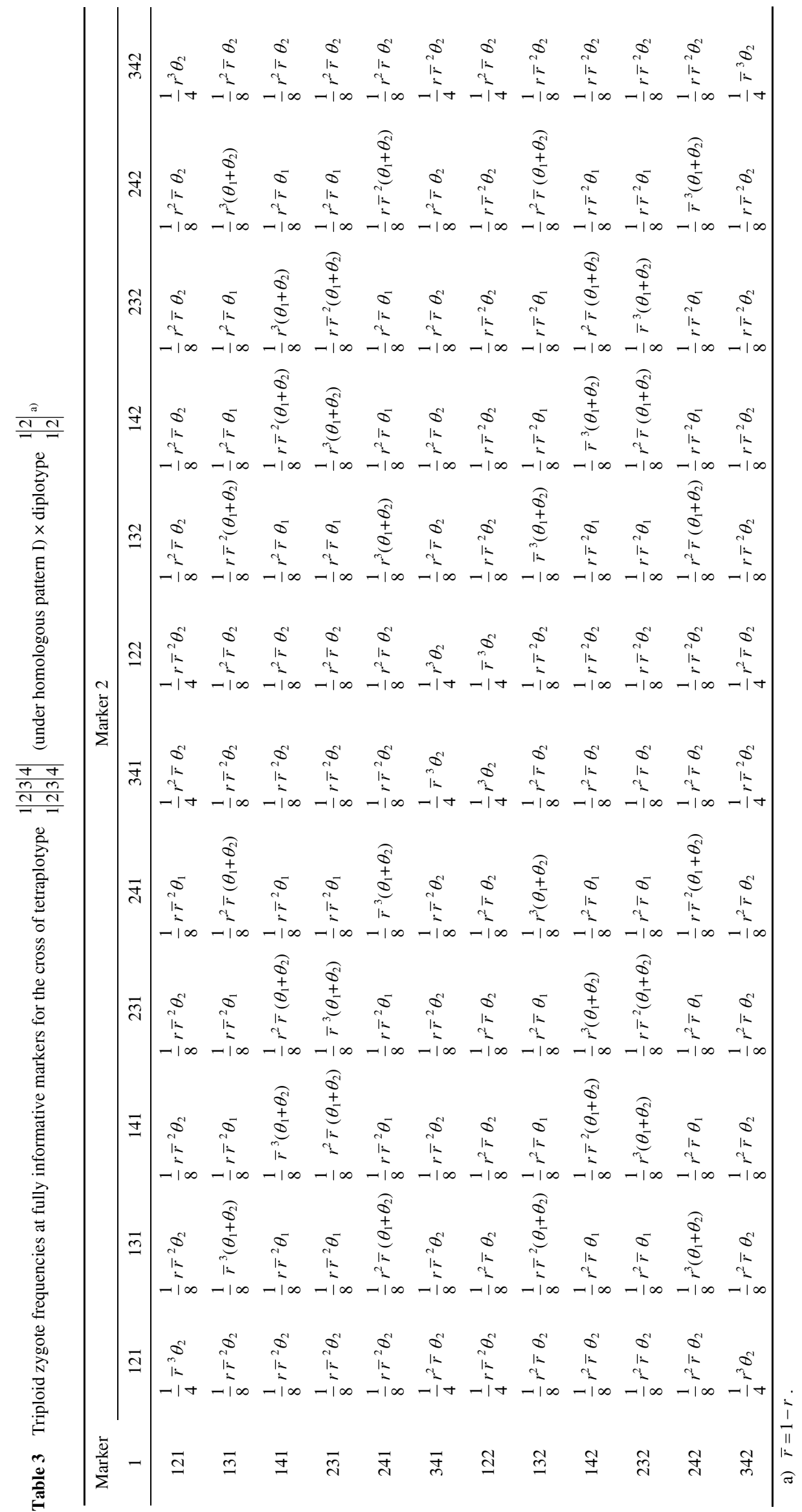


Table 4 Triploid zygote frequencies at partially informative markers for the cross of tetraplotype \begin{tabular}{lll|l|l|}
1 & 2 & 2 & 2 \\
2 & 3 & 3
\end{tabular} \mid (under homologous pattern I) $\times$ diplotype $\begin{aligned} & 1 \\
& 1\end{aligned}\left|\begin{array}{l}2 \\
2\end{array}\right|$

\begin{tabular}{|c|c|c|c|c|c|c|c|c|}
\hline \multirow{2}{*}{ Marker 1} & \multicolumn{8}{|c|}{ Marker 2} \\
\hline & 121 & 131 & 231 & 331 & 122 & 132 & 232 & 332 \\
\hline 12 & $\frac{1}{4}(1-r)^{2} \theta_{2}$ & $\frac{1}{4}(1-r)^{2}\left(\theta_{1}+\theta_{2}\right)$ & $\frac{1}{4} r(1-r)\left(\theta_{1}+\theta_{2}\right)$ & $\frac{1}{4} r(1-r) \theta_{2}$ & $\frac{1}{4} r(1-r) \theta_{2}$ & $\frac{1}{4} r(1-r)\left(\theta_{1}+\theta_{2}\right)$ & $\frac{1}{4} r^{2}\left(\theta_{1}+\theta_{2}\right)$ & $\frac{1}{4} r^{2} \theta_{2}$ \\
\hline 22 & $\frac{1}{4} r(1-r) \theta_{2}$ & $\frac{1}{4} r(1-r)\left(\theta_{1}+\theta_{2}\right)$ & $\frac{1}{4}(1-r)^{2}\left(\theta_{1}+\theta_{2}\right)$ & $\frac{1}{4}(1-r)^{2} \theta_{2}$ & $\frac{1}{4} r^{2} \theta_{2}$ & $\frac{1}{4} r^{2}\left(\theta_{1}+\theta_{2}\right)$ & $\frac{1}{4} r(1-r)\left(\theta_{1}+\theta_{2}\right)$ & $\frac{1}{4} r(1-r) \theta_{2}$ \\
\hline 12 & $\frac{1}{4} r(1-r) \theta_{2}$ & $\frac{1}{4} r(1-r)\left(\theta_{1}+\theta_{2}\right)$ & $\frac{1}{4} r^{2}\left(\theta_{1}+\theta_{2}\right)$ & $\frac{1}{4} r^{2} \theta_{2}$ & $\frac{1}{4}(1-r)^{2} \theta_{2}$ & $\frac{1}{4}(1-r)^{2}\left(\theta_{1}+\theta_{2}\right)$ & $\frac{1}{4} r(1-r)\left(\theta_{1}+\theta_{2}\right)$ & $\frac{1}{4} r(1-r) \theta_{2}$ \\
\hline 22 & $\frac{1}{4} r^{2} \theta_{2}$ & $\frac{1}{4} r^{2}\left(\theta_{1}+\theta_{2}\right)$ & $\frac{1}{4} r(1-r)\left(\theta_{1}+\theta_{2}\right)$ & $\frac{1}{4} r(1-r) \theta_{2}$ & $\frac{1}{4} r(1-r) \theta_{2}$ & $\frac{1}{4} r(1-r)\left(\theta_{1}+\theta_{2}\right)$ & $\frac{1}{4}(1-r)^{2}\left(\theta_{1}+\theta_{2}\right)$ & $\frac{1}{4}(1-r)^{2} \theta_{2}$ \\
\hline
\end{tabular}

Table 5 Marker types for the citrus cross between allotetraploid male (HR) and a diploid hybrid female

\begin{tabular}{|c|c|c|c|c|c|c|c|c|}
\hline \multirow{2}{*}{ Type } & \multicolumn{2}{|c|}{$\mathrm{Hm}$} & \multicolumn{2}{|c|}{ RL } & \multicolumn{2}{|c|}{ Tetraploid HR } & \multicolumn{2}{|c|}{ Diploid IB } \\
\hline & Pheno & Geno & Pheno & Geno & Pheno & Geno & Pheno & Geno \\
\hline \multirow[t]{2}{*}{1} & 1 & 11 & 0 & 00 & 1 & 1100 & 0 & 00 \\
\hline & & 10 & & & & 1000 & & \\
\hline 2 & 0 & 00 & 1 & 11 & 1 & 0011 & 0 & 00 \\
\hline 3 & 1 & 10 & 1 & 10 & 1 & 1010 & 0 & 00 \\
\hline \multirow[t]{2}{*}{4} & 1 & 11 & 0 & 00 & 1 & 1100 & 1 & 10 \\
\hline & & 10 & & & & 1000 & & \\
\hline 5 & & & & 10 & & 0010 & & \\
\hline 6 & 1 & 10 & 1 & 10 & 1 & 1010 & 1 & 10 \\
\hline 7 & 0 & 00 & 0 & 00 & 0 & 0000 & 1 & 10 \\
\hline
\end{tabular}

assume that preference pairings take place between the two Hm chromosomes and between the two RL chromosomes. The first three two-marker genotypes should each have a unique diplotype, respectively, expressed as

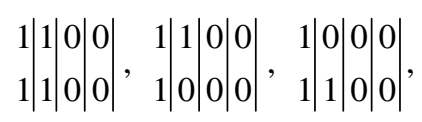

which produces diploid gametes with frequencies, respectively, as follows

\begin{tabular}{c|cc} 
& $1_{-}$ & 00 \\
\hline $1_{-}$ & $\left(r^{2}-\frac{1}{2}(1-r)^{2}+2(1-r)\right) \theta_{2}+\theta_{1}$ & $\left(\frac{1}{2} r^{2}+r(1-r)\right) \theta_{2}$, \\
00 & $\left(\frac{1}{2} r^{2}+r(1-r)\right) \theta_{2}$ & $\frac{1}{2}(1-r)^{2} \theta_{2}$
\end{tabular}

\begin{tabular}{c|cc} 
& $1_{-}$ & 00 \\
\hline $1_{-}$ & $\left(1-\frac{1}{2} r\right) \theta_{2}+\frac{1}{2} \theta_{1}$ & $\frac{1}{2} \theta_{1}+\left(\frac{1}{2}+\frac{1}{2} r\right) \theta_{2}$, \\
00 & $\frac{1}{2} r \theta_{2}$ & $\frac{1}{2}(1-r) \theta_{2}$
\end{tabular}

\begin{tabular}{c|cc}
$1-$ & 00 \\
\hline $1_{-}$ & $\left(1-\frac{1}{2} r\right) \theta_{2}+\frac{1}{2} \theta_{1}$ & $\frac{1}{2} r \theta_{2}$ \\
00 & $\frac{1}{2} \theta_{1}+\left(\frac{1}{2}+\frac{1}{2} r\right) \theta_{2}$ & $\frac{1}{2}(1-r) \theta_{2}$
\end{tabular}

The last genotype may have two possible diplotypes, i.e.,

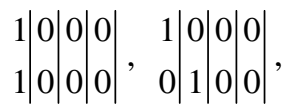


with a probability of $q$ and 1-q, respectively. Each diplotype may produce different frequencies of diploid gametes, but their overall frequencies, weighted by diplotype probabilities, are given as

$$
\begin{array}{c|cc} 
& 1_{-} & 00 \\
\hline 1_{-} & \frac{1}{2}(1-r) q+\left(\frac{1}{2} r \theta_{1}+\frac{1}{2} \theta_{2}\right)(1-q) & \frac{1}{2} r q+\left(\frac{1}{2}(1-r) \theta_{1}+\frac{1}{2} \theta_{2}\right)(1-q) \\
00 & \frac{1}{2} r q+\left(\frac{1}{2}(1-r) \theta_{1}+\frac{1}{2} \theta_{2}\right)(1-q) & \frac{1}{2}(1-r) q+\left(\frac{1}{2} r \theta_{1}+\frac{1}{2} \theta_{2}\right)(1-q)
\end{array} .
$$

Based on matrices (8), (9), (10), and (12), we implement the EM algorithm to estimate the recombination fraction and preferential pairing factor. For matrix (12), we will also need to estimate diplotype probability $q$. An optimal marker genotype combination can be selected from the larger likelihood.

We use the preferential pairing model to analyze some of the dominant markers that belong to types 1-3 of Table 5 . The recombination fraction between different markers and the preferential pairing factor $(p)$ were estimated simultaneously using the model. The estimated recombination fractions were converted to genetic distances using Kosambi's map function. A total of seven markers were localized in the same linkage group with genetic distances shown in Figure 1. The estimate of $p$ was quite low, suggesting that the allotetraploid parent (HR) tends to pair their chromosomes with an equal likelihood during meiosis.

\subsection{Computer simulation}

We performed computer simulation to test the statistical behavior of the model. Because the first type of markers can

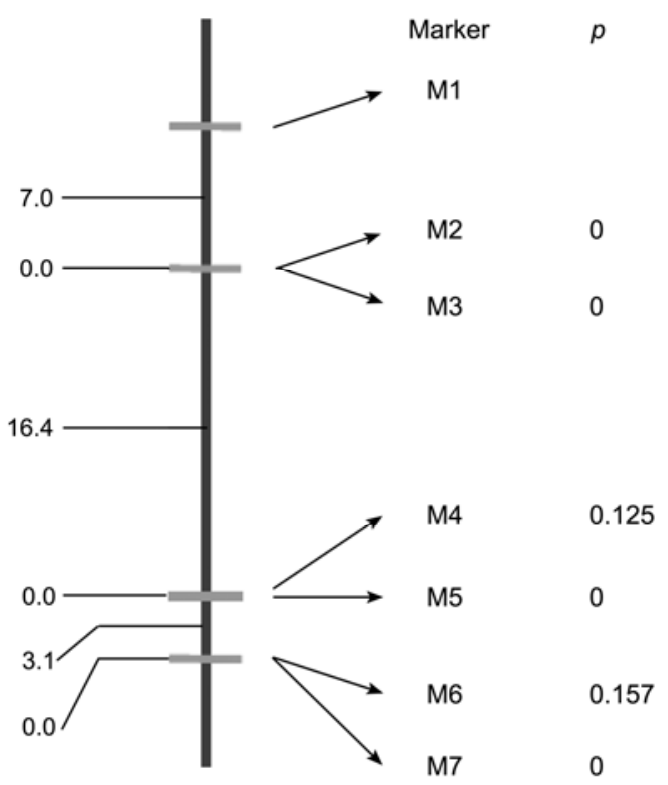

Figure 1 Linkage groups composed of dominant markers that belong to types 1-3 of Table 5 in citrus triploid hybrids. Estimates of map distances and the preferential pairing factor $(p)$ using a pair of markers were shown. be analyzed by a general backcross model [9], our simulation will focus on linkage analysis of the next two types of markers. As a demonstration, we design two representative mating designs for pseudotest markers under the gametic model:

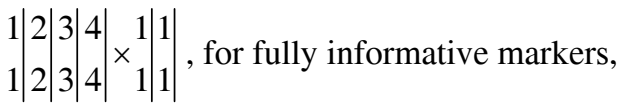

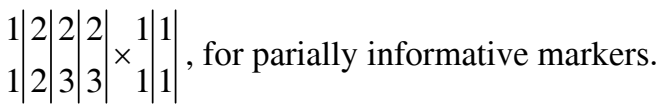

Genotype data were simulated by considering the following four scenarios: (1) no preferential pairing $(p=0)$ and low linkage $(r=0.3)$, (2) no preferential pairing $(p=0)$ and high linkage $(r=0.05)$, (3) high preferential pairing $(p=0.4)$ and low linkage $(r=0.3)$, and (4) high preferential pairing $(p=0.4)$ and high linkage $(r=0.05)$. Each scenario includes different sample sizes, 100, 200, and 400. The simulated data were analyzed by the triploid model incorporating preferential pairing.

For fully informative markers, the preferential pairing model provided reasonable estimates of both the recombination fraction and preferential pairing factor $(p)$ under the four scenarios even if a small sample size (100) was used (Table 6). When there is no preferential pairing, the model still found a small $p$ value which is false positive. However, the good thing is that false positive rates are reasonably low $(<0.10)$. If preferential pairing occurs, the model displays sufficient power to detect this phenomenon. As expected, the precision of parameter estimates by the new model and its power and false positive rates can be improved when sample size increases (results not shown).

Compared with fully informative markers, the estimation precision of parameters for partially informative markers is reduced due to a less amount of informativeness (Table 7). For a particular mating design (14), different results are obtained from homologous patterns I and II or III. The estimation precision of $p$, power of $p$ detection and false positive rates are much worse under pattern II or III than pattern I. This may be due to the fact that chromosomes 3 and 4 carry the same alleles at these two markers and, thus, they tend to be more homologous. All these can be improved by doubling sample size to 200 (data not shown).

An additional simulation was carried out to judge whether 
Table 6 Maximum likelihood estimates of the recombination fraction ( $r$ )

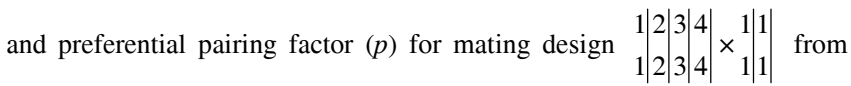
1000 simulation replicates under different simulation scenarios in a mapping population of size $100^{\text {a) }}$

\begin{tabular}{|c|c|c|c|c|c|c|}
\hline \multirow{2}{*}{ Scenario } & \multicolumn{2}{|c|}{ True } & \multicolumn{2}{|c|}{ Estimation } & \multirow{2}{*}{ Power } & \multirow{2}{*}{ FPR } \\
\hline & $p$ & $r$ & $p$ & $r$ & & \\
\hline \multirow[t]{2}{*}{1} & 0 & 0.05 & 0.0342 & 0.0506 & - & 0.081 \\
\hline & & & $(0.0502)$ & $(0.0155)$ & & \\
\hline \multirow[t]{2}{*}{2} & 0 & 0.30 & 0.0269 & 0.2982 & - & 0.096 \\
\hline & & & $(0.0383)$ & $(0.0320)$ & & \\
\hline \multirow[t]{2}{*}{3} & 0.4 & 0.05 & 0.4009 & 0.0502 & 1 & - \\
\hline & & & $(0.0644)$ & $(0.0165)$ & & \\
\hline \multirow[t]{2}{*}{4} & 0.4 & 0.30 & 0.4018 & 0.2993 & 1 & - \\
\hline & & & $(0.0529)$ & $(0.0316)$ & & \\
\hline
\end{tabular}

a) Numbers in parentheses are the standard errors of the estimates. The power for $p$ identification by the new model and its false positive rates (FPR) are also shown.

the new model can correctly discern homologous patterns. We used homologous pattern I to simulate fully informative marker data under four simulation scenarios and then analyzed the data with the model derived from different homologous patterns. It was found that when $p=0.5$, the model has about 0.70 probability to correctly identify the homologous pattern. This probability will increase to about one when $p$ increases to 0.30 . A similar conclusion was detected for partially informative markers simulated from mating design (14).

The same simulation design was used to analyze intercross marker data which were then analyzed by the zygotic model. In general, similar results were found for the precision of parameter estimation by the zygotic model as well as its power and false positive rates.

\section{Discussion}

Chromosomal pairings in bivalent polyploids depend on the homology between the genomes involved, with a preference in pairing between homologous over homoeologous chromosomes [22-24]. Wu et al. [12] incorporated this so-called preferential pairing factor into a linkage model in polyploids by better capturing the cytological pairing mechanisms for meiotic configurations and inheritance in polyploids. This incorporation is shown to improve the accuracy of the estimation of the recombination fraction for some particular marker types in bivalent polyploids. The estimation of the preferential pairing factor helps to understand the cytological mechanisms of polyploids and relatedness of different genomes involved [25-27].

The development of the model presented in this article was motivated from segregating triploid populations detected in many plant species $[1,6,7,15]$. We considered a case in which triploids were generated from crossing a tetraploid and diploid. The tetraploid parent was assumed to follow bivalent meiosis during which more homologous chromosomes pair more likely than less homologous chromosomes. We derived gametic and zygotic models for pseudotest markers and intercross markers, respectively. For both models, we first derived a general model for analyzing the linkage between fully informative markers and then extended the model to accommodate the segregating pattern of other partially informative markers. Linkage analysis of less informative markers needs a special treatment by developing the sophisticated EM algorithm. We performed simulation studies to validate the usefulness of our model for estimating the linkage and preferential pairing factor. The model was used to reanalyze a published data set for a triploid progeny in Citrus. Several dominant markers were detected to be linked on a group. More importantly, by estimating the

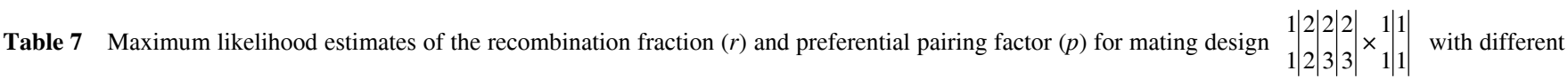
homologous patterns from 1000 simulation replicates under different simulation scenarios in a mapping population of size $100^{\mathrm{a})}$

\begin{tabular}{|c|c|c|c|c|c|c|c|c|c|c|}
\hline \multirow{3}{*}{$\begin{array}{l}\text { Sce- } \\
\text { nario }\end{array}$} & \multicolumn{2}{|c|}{ True } & \multicolumn{4}{|c|}{ Homologous pattern I } & \multicolumn{4}{|c|}{ Homologous pattern II or III } \\
\hline & \multirow{2}{*}{$p$} & \multirow{2}{*}{$r$} & \multicolumn{2}{|c|}{ Estimation } & \multirow{2}{*}{ Power } & \multirow{2}{*}{ FPR } & \multicolumn{2}{|c|}{ Estimation } & \multirow{2}{*}{ Power } & \multirow{2}{*}{ FPR } \\
\hline & & & $p$ & $r$ & & & $p$ & $r$ & & \\
\hline \multirow[t]{2}{*}{1} & 0 & 0.05 & 0.0031 & 0.0495 & - & 0.097 & 0.0079 & 0.0496 & - & 0.109 \\
\hline & & & $(0.0961)$ & $(0.0213)$ & & & $(0.1149)$ & $(0.0211)$ & & \\
\hline \multirow[t]{2}{*}{2} & 0 & 0.30 & 0.0056 & 0.3015 & - & 0.080 & 0.0073 & 0.2972 & - & 0.108 \\
\hline & & & $(0.0971)$ & $(0.0454)$ & & & $(0.1060)$ & $(0.0456)$ & & \\
\hline \multirow[t]{2}{*}{3} & 0.4 & 0.05 & 0.3962 & 0.0488 & 0.989 & - & 0.4068 & 0.0494 & 0.580 & - \\
\hline & & & $(0.0658)$ & $(0.0224)$ & & & $(0.1960)$ & $(0.0226)$ & & \\
\hline \multirow[t]{2}{*}{4} & 0.4 & 0.30 & 0.4049 & 0.3024 & 1 & - & 0.4003 & 0.3022 & 0.564 & - \\
\hline & & & $(0.0667)$ & $(0.0446)$ & & & $(0.1935)$ & $(0.0437)$ & & \\
\hline
\end{tabular}

a) Numbers in parentheses are the standard errors of the estimates. The power for $p$ identification by the new model and its false positive rates (FPR) are also shown. 
preferential pairing factor, we have for the first time reported the extent of homologous paring between different chromosomes of different Citrus species.

Our model was based on two-point linkage analysis. It is important to extend it to a three-point analysis because a multi-locus model will provide more information about meiotic functions and mechanisms. For example, a threepoint analysis allows the test and estimation of genetic interferences and their effects on the recombination events. In statistics, a three-point analysis helps to increase the power of linkage detection and the precision of parameter estimation especially when markers are dominant. A three-point analysis is also a foundation of interval mapping for quantitative trait loci affecting complex phenotypes. A QTL mapping model that incorporates the preferential pairing factor will provide a new powerful tool to study the genetics of polyploids.

The model for bivalent tetraploids can be extended, with no theoretical difficulty, to handle bivalent polyploids of an arbitrary ploidy level, such as hexaploid, octoploids and dexaploids [28]. A key issue for modeling these high-ploidy polyploids is how to characterize the preferential pairing factor. Sybenga [23] proposed a genetic model for determining preferential chromosomal pairings in hexaploids. Our model assumes that marker segregation follows Mendel's law, but this assumption may be violated. In Wu et al. [9], models for linkage analysis with distorted or misclassified markers are described for diploid species, which should be extended to precisely identify the linkage in polyploid species. Also, given the widespread occurrence of polyploidy in nature, it is crucial to study the genome organization of polyploids based on the linkage disequilibria of molecular markers. Hou et al. [29] proposed a statistical design that integrates the linkage, linkage disequilibrium, and inbreeding rate to analyze genetic diversity and mating behavior in diploid populations. A similar integrative model can be developed for polyploid populations by implementing our linkage analysis model in Hou et al.'s design. A powerful statistical method for linkage analysis and linkage disequilibrium analysis using molecular markers, increasingly available for polyploids, provides an important step towards studying the genetic basis of quantitative variation in these underrepresented but important species.

This work was supported by Fundamental Research Funds for the Central Universities (YX2011-30, YX2010-30, BLYX200924 and JD2010-5), Special Fund for Forestry-Scientific Research in the Public Interest (201004017), National Natural Science Foundation of China (61170268), China Agricultural Research System-CARS; NSF/IOS-0923975 and Changjiang Scholars Award; and "Thousand-person Plan" Award.

1 Zhu Z T, Kang X Y, Zhang Z Y. Studies on selection of natural triploids of Populus tomentosa. Sci Silv Sin, 1998, 34: 22-31
2 Benson M K, Einspahr D W. Early growth of diploid and triploid hybrid aspen. Forest Sci, 1967, 13: 150-155

3 Hilu K W. Polyploidy and the evolution of domesticated plants. Am J Bot, 1993, 80: 1491-1499

4 Thomas T D, Chaturvedi R. Endosperm culture: A novel method for triploid plant production. Plant Cell Tiss Org Cult, 2008, 93: 1-14

5 Nilsson-Ehle $\mathrm{H}$. Uber eine in der natur gefundene gigantsform von Populus tremula. Hereditas, 1936, 21: 379-382

6 Perala D A. Data on file. USDA Forest Service, North Central Forest Experiment Station, Grand Rapids, MN, 1989

7 Ewald D, Ulrich K, Naujoks G, et al. Induction of tetraploid poplar and black locust plants using colchicine: Chloroplast number as an early marker for selecting polyploids in vitro. Plant Cell Tiss Organ Cult, 2009, 99: 353-357

8 d'Erfurth I, Jolivet S, Froger N, et al. Turning meiosis into mitosis. PLoS Biol, 2009, 7: e1000124

9 Wu R L, Ma C X, Casella G. Statistical Genetics of Quantitative Traits: Linkage, Maps, and QTL. New York: Springer-Verlag, 2007

10 Luo Z W, Hackett C A, Bradshaw J E, et al. Construction of a genetic linkage map in tetraploid species using molecular markers. Genetics, 2001, 157: 1369-1385

11 Wu S S, Wu R L, Ma C X, et al. A multivalent pairing model of linkage analysis in autotetraploids. Genetics, 2001, 159: 2000-2012

12 Wu R L, Ma C X, Casella G. A bivalent polyploid model for linkage analysis in outcrossing tetraploid species. Theor Pop Biol, 2002, 62: 129-151

13 Johnsson H. The triploid progeny of the cross diploid-tetraploid Populus tremula. Hereditas, 1945, 31: 411-440

14 Perala D A. Populus trembuloides Michx. Quaking aspen. In: Burns R M, Honkala N B, eds. Silvics of North America. Volume 2, Hardwoods. USDA Forest Service Agricultural Handbook 654. Washington DC: USDA Forest Service, 1990. 555-569

15 Yin H L, Deng X X. Inheritance analysis of sexual hybrids derived from somatic citrus using RAPD markers. Sci China Ser C-Life Sci, 2007, 37: 42-51

16 Wu R L, Gallo-Meagher M, Littell R C, et al. A general polyploid model for analyzing gene segregation in outcrossing tetraploid species. Genetics, 2001, 159: 869-882

17 Wu R L, Ma C X. A general framework for statistical linkage analysis in multivalent tetraploids. Genetics, 2005, 170: 899-907

18 Stebbins G L. Variation and Evolution in Plants. New York: Columbia University Press, 1950

19 Sybenga J. The quantitative analysis of chromosome pairing and chiasma formation based on the relative frequencies of MI configurations. VII. Autotetraploids. Chromosoma, 1975, 50: 211-222

20 Sybenga J. Mathematical models for estimating preferential pairing and recombination in triploid hynrids. Genome, 1988, 30: 745-757

21 Kepiro J L, Roose M L. AFLP markers closely linked to a major gene essential for nucellar embryony (apomixis) in Citrus maxima $\times$ Poncirus trifoliata. Tree Genet Genom, 2009, 6: 1-11

22 Jackson R C, Casey J. Cytogenetic analysis of autopolyploids: Models and methods for triploids to octoploids. Am J Bot, 1982, 69: 487-501

23 Sybenga J. What makes homologous chromosomes find each other in meiosis? A review and a hypothesis. Chromosoma, 1999, 108: 209-219

24 Jackson R C, Jackson J W. Gene segregation in autotetraploids: Prediction from meiotic configurations. Am J Bot, 1996, 83: 673-678

25 Ramsey J, Schemske D W. Pathways, mechanisms, and rates of polyploid formation in flowering plants. Ann Rev Ecol Syst, 1998, 29: 467-501

26 Otto S P, Whitton J. Polyploid incidence and evolution. Ann Rev Genet, 2000, 34: 401-437

27 Soltis P S, Soltis D E. The role of genetic and genomic attributes in the success of polyploids. Proc Natl Acad Sci USA, 2000, 97: 7051-7057

28 da Silva J, Sorrells M E, Burnquist W L, et al. RFLP linkage map and genome analysis of Saccharum spontaneum. Genome, 1993, 36: 782-791

29 Hou W, Liu T, Li Y, et al. Multilocus genomics of outcrossing populations. Theor Pop Biol, 2009, 76: 68-76

Open Access This article is distributed under the terms of the Creative Commons Attribution License which permits any use, distribution, and reproduction in any medium, provided the original author(s) and source are credited. 